\title{
Sul concetto di fondamento in Karl Leonhard Reinhold (1789-1792). Sei tesi sul passaggio dalla Critica della ragione alla Filosofia elementare
}

On Karl Leonhard Reinhold's Concept of Ground (1789-1792).

Six Thesis on the Transition from the Critique of Reason to the Elementary Philosophy

Sobre o conceito de fundamento em Karl Leonhard Reinhold (1789-1792).

Seis teses sobre a passagem da Crítica da razão à Filosofia elementar

\section{Faustino Fabbianelli*}

Università di Parma, Parma, Italia 


\section{Riassunto}

Il concetto del fondamento reinholdiano riprende solo nominalmente il corrispondente concetto kantiano. Se è vero che in entrambi i casi ciò che fonda il fondato è quell'elemento che pone il diverso da sé in maniera determinata, resta da stabilire come tale rapporto fondativo debba essere compreso. Per Kant si mostra al proposito fondamentale la questione della sintesi che permette non soltanto di distinguere tra la conoscenza matematica e la conoscenza filosofica, ma anche di fissare l'impossibilità, per la filosofia, di partire da definizioni. Reinhold è dell'avviso che una Filosofia senza appellativi, come intende essere la Filosofia elementare, possa andare oltre i limiti imposti da Kant alla conoscenza filosofica nella misura in cui è in grado di fondare il sapere su fondamenti che, in quanto assolutamente determinati, spiegano l'esperienza in base alla loro indubitabilità. Il tema della notio ovvero della omnimoda determinatio, intorno al quale era ruotata gran parte della discussione della scolastica leibniziano-wolffiana in relazione alla definizione e alla conoscibilità di una cosa, viene ripreso da Reinhold all'interno di una filosofia trascendentale che va oltre Kant e che apre le porte ai grandi sistemi della filosofia classica tedesca.

Parole-chiave: Reinhold. Kant. Fichte. Fondamento. Sintesi. Matematica.

\section{Resumo}

O conceito de fundamento reinholdiano retoma nominalmente o correspondente conceito kantiano. Se é verdade que em ambos os casos o que funda é aquele elemento que põe o diverso por si de modo determinado, temos que estabelecer como esta relação fundacional tenha que ser compreendida. Para Kant aparece fundamental a questão da síntese, que permite, não apenas diferenciar entre consciência matemática e filosófica, mas também fixar a impossibilidade, para a filosofia, de partir de definições. Reinhold acha que uma filosofia sem apelações, como pretende ser a filosofia elementar, possa ir além dos limites impostos por Kant ao conhecimento filosófico, na medida em que ele é capaz de fundar o saber em fundamentos que, como são absolutamente determinados, explicam a experiência em base ao fato de que não se pode duvidar sobre eles. O tema da notio, ou seja da omnimoda determinatio, em torno ao qual girava boa pare da discussão da escolástica leibniziano-wollfiana no que diz respeito à definição e à cognoscibilidade de uma coisa, é retomado 
por Reinhold no âmbito de uma filosofia transcendental que vai além de Kant e que abre as portas aos grandes sistemas da filosofia clássica alemã.

Palavras-chave: Reinhold. Kant. Fichte. Fundamento. Síntese. Matemática.

\section{Abstract}

Reinhold's concept of ground only nominally refers to the corresponding Kantian concept. If it is true that in both cases what founds the founded is that element that sets the different from itself in a determined manner, it remains to be defined how this foundational relationship must be understood. For Kant, the question of synthesis allows not only to distinguish between mathematical and philosophical knowledge, but also to establish the impossibility for philosophy to start from definitions. Reinhold thinks that a philosophy without epithets, as it intends to be the Elementary Philosophy, can go beyond the limits imposed by Kant to philosophical knowledge by founding cognition on grounds which, as absolutely determined, explain experience through their indubitability. The theme of the notio or the omnimoda determinatio, around which a large part of the discussion of Leibniz-Wolff's scholasticism was revolved in relation to the definition and the knowability of a thing, is taken up by Reinhold within a transcendental philosophy that goes beyond Kant and opens the doors to the great systems of classical German philosophy.

Keywords: Reinhold. Kant. Fichte. Ground. Synthesis. Mathematics.

\section{Tesi 1: La polisemia del concetto di «fondamento» reinholdiano è riconducibile ad un significato unitario.}

In una lettera del 12 maggio 1789 indirizzata a Reinhold Kant, confrontandosi con il leibniziano Johann August Eberhard, afferma che fondamento (Grund) è in generale ciò «mediante cui qualcos'altro (qualcosa di diverso) viene posto in modo determinato (qvo posito determinate ponitur aliud)» (RKA 2, p. 97). Il rapporto tra il fondamento 
e la sua consequenza (Folge) può tuttavia essere compreso o in senso logico - Kant parla di differenza nel «modo della rappresentazione» - o in senso reale - Kant dice «nell'oggetto stesso» (RKA 2, p. 98). Quando ad esempio ci si riferisce alla relazione tra il concetto di esteso e il concetto di divisibile, si intende un rapporto che contiene una mera differenza logica tra due momenti, la quale non esclude affatto la loro identità: «la divisibilità è infatti davvero compresa nel concetto dell'estensione» (RKA 2, p. 98). Ciò non avviene invece nella relazione del fondamento sintetica, nella quale si va alla ricerca di un fondamento reale (Realgrund) di una certa cosa. Se nel primo caso si astrae dal contenuto della relazione - e infatti la logica afferma che tutti i giudizi assertori devono avere un fondamento, disinteressandosi tuttavia di ciò che costituisce effettivamente la differenza reale, vale a dire tra una cosa e il suo fondamento rispetto al contenuto - , nel secondo caso, che è poi quello che riguarda propriamente i giudizi sintetici, il rapporto tra il fondamento e la sua conseguenza viene stabilito sulla base del contenuto che li unisce. Il principio di ragion sufficiente può pertanto avere sia un valore meramente logico e riguardare solo «fondamenti analitici» - e solo in tal caso il principio del fondamento (Satz des Grundes) può essere fatto derivare dal principio di (non) contraddizione - , oppure può avere un valore reale nel senso che tenendo conto del contenuto della relazione esso si riferisce a fondamenti reali. Kant aggiunge che poiché la sintesi a priori può essere tanto matematica quanto filosofica, anche il fondamento reale deve essere inteso in maniera duplice: come formale - quando ad esempio «i lati del triangolo contengono il fondamento degli angoli», oppure come materiale, quando esso concerne l'esistenza delle cose e «fa sì che ciò che lo contiene venga chiamato causa» (RKA 2, p. 98) ${ }^{1}$.

La concettualità utilizzata da Kant viene ripresa, talvolta alla lettera, nella recensione del «Philosophisches Magazin» di Eberhard che Reinhold pubblica sulla «Allgemeine Literatur-Zeitung» (n. 174-176, 11-13 giugno 1789). La si ritrova però anche nelle opere a stampa e nel Corso di Logica e Metafisica che Reinhold tiene a Jena nel semestre

Sul concetto di Grund in Kant, anche in relazione a Reinhold, si veda TEICHNER, 1976. 
estivo del $1792^{2}$. In generale, si può affermare che il fondamento ha per Reinhold, come del resto già per Kant, una rilevanza filosofica in particolare nella sua accezione reale e quindi in relazione all'ambito del sintetico e non dell'analitico.

Se ora si guarda un po' meglio alle occorrenze del concetto di fondamento in Reinhold, ci si trova di fronte ad una polisemia (fondamento esplicativo (Erklärungsgrund), ragione sufficiente (zureichender Grund), fondamento cagionante e determinante (veranlassender/ bestimmender Grund) etc.) che solo prima facie ne esclude l'unitarietà. A ben vedere, infatti, c'è un modo in cui Reinhold determina il concetto di fondamento il quale unisce, come loro momento qualificante, le diverse occorrenze in cui esso si presenta. Come si vedrà, è proprio con questo significato principale che si va oltre la corrispondente teoria di Kant. Cercherò di mostrare ciò facendo riferimento in particolare a tre differenti significati del fondamento (tesi 3-5).

Al fine di introdurre la questione, è opportuno, in linea preliminare, ritornare al sopra menzionato Corso di Logica e Metafisica nel quale Reinhold distingue il concetto del fondamento in rapporto alla perfezione (Vollkommenheit), alla distinzione (Deutlichkeit) e alla determinatezza (Bestimmtheit) della conoscenza. Laddove la conoscenza comune (gemein) è «disordinata e incompleta, dal momento che sorge soltanto in maniera casuale», la conoscenza filosofica non deve «in nessun caso essere rapsodica» (RGS 12, p. 25). Ed essa può non essere rapsodica nella misura in cui le sue dimostrazioni logicamente corrette consistono «nell'esposizione precisa e completa dei fondamenti» su cui «si fonda la necessità di un giudizio» (RGS 12, p. 109).

In che misura tuttavia i fondamenti di una prova possono essere esposti in modo preciso e completo? La parte del Corso dedicata alla logica afferma al riguardo che una dimostrazione scientifica necessita di definizioni e di suddivisioni che possano essere intese come verità fondamentali. Se si vuole ad esempio provare che l'anima è immortale, è necessario prendere le mosse da una definizione corretta della sua

2 Se ne possiede una versione negli appunti che lo studente Johann Smidt redige in classe. Sulla datazione del Corso di Logica si veda l'Introduzione a RGS 12, p. VII-XLIX. 
natura. Ed una tale definizione potrà essere corretta soltanto se «è assolutamente determinata in tutte le sue note (Merkmale)» (RGS 12, p. 112). Se così non fosse, potrebbe infatti verificarsi di addurre nella dimostrazione un fondamento di determinazione che è falso nella misura in cui viene fatto derivare «da una nota superflua della definizione» (RGS 12, p. 112). Può dunque essere considerato autentico soltanto quel fondamento correlato ad una nota corretta. Le prove dell'immortalità dell'anima finora condotte sono secondo Reinhold sbagliate in quanto «contengono la nota della cosa in sé che falsifica necessariamente tutto» (RGS 12, p. 112).

\section{Tesi 2: Qualcosa può essere considerato un fondamento soltanto per le note che lo costituiscono.}

L'appena rilevato nesso intrinseco tra il fondamento e le sue note costituisce l'aspetto caratteristico della dottrina reinholdiana. Se infatti si domanda perché qualcosa possa essere considerato un fondamento, si deve con Reinhold rispondere che sono le sue note determinate che lo rendono fondamento di qualcos'altro. Il già richiamato rapporto tra fondamento (Grund) e conseguenza (Folge) chiarisce questo punto in modo evidente. Certe note di un certo concetto fondano la possibilità ovvero la realtà di altre note del medesimo concetto e introducono pertanto la relazione di fondamento e conseguenza. Detto con le parole del Corso di Logica: «Nella proposizione "l'uomo è un animale razionale", le note sulle quali riposa il fondamento di tutte le rimanenti note del concetto di umanità sono l'animalità e la ragione» (RGS 12, p. 57). Qualcosa può dunque essere considerato il fondamento di qualcos'altro se contiene in sé una o più note che spiegano il concetto del fondato nel suo essere determinato e posto.

Questa concezione si inserisce nella dottrina più generale per la quale scopo della filosofia è raggiungere concetti distinti, assumere cioè tutte le note essenziali che appartengono ad un concetto e di lasciare da parte tutte quelle che non gli appartengono (RGS 12, p. 28). Solo in tale modo diventa infatti possibile evitare i malintesi 
che hanno compromesso le discussioni filosofiche. Reinhold riprende qui chiaramente il tema della notio ovvero della omnimoda determinatio che aveva giocato un ruolo determinante all'interno della filosofia leibniziano-wolffiana in relazione alla definizione e alla conoscibilità di una $\operatorname{cosa}^{3}$. Proprio una tale concezione sta alla base della distanza che sussiste tra la dottrina del fondamento kantiana e quella reinholdiana. Si potrebbe anche dire che la relazione che in Kant unisce il concetto del fondamento con la domanda sui giudizi sintetici a priori si lega in Reinhold alla questione intorno alla definibilità di una certa cosa come fondamento di un'altra cosa a partire dalle note che le appartengono in maniera essenziale. Alla questione della possibilità dei giudizi sintetici a priori si affianca, fino a sostituirsi ad essa, la domanda sull'analisi dei concetti fondamentali del sapere ${ }^{4}$.

Se ora ci si domanda perché un tale passo avvenga, diventa necessario fare riferimento, in particolare, alla strategia speculativa di cui esso è parte integrante e per la quale la domanda sul fondamento ha una relazione privilegiata con l'edificazione di una filosofia del fondamento (Grundsatzphilosophie) all'interno della quale il tema kantiano della sintesi a priori assume un ruolo secondario. Nella Fundamentschrift si legge al proposito che la Filosofia elementare è in grado di avvalersi di definizioni rigorose sulle quali fondare il sistema del sapere. Laddove Kant ritiene che le definizioni - intese come le esposizioni originariamente dettagliate di una cosa entro i suoi limiti (Critica della ragion pura, B 755) - possano darsi soltanto nella matematica, dal momento che solo in essa si può essere sicuri dell'adeguatezza del concetto con l'oggetto mediante l'intuizione -, Reinhold, pur trovandosi d'accordo con Kant sul fatto che definire significa esporre dettagliatamente, cioè in modo chiaro e sufficiente le note del concetto della cosa, ritiene che una tale richiesta possa essere soddisfatta unicamente dalla Filosofia elementare. Essa è infatti in grado di avvalersi di definizioni che sono vere in primo luogo perché la loro correttezza dipende dalla correttezza di «un'unica, suprema e ultima definizione le

3 Si veda su questo punto REIMARUS, 1766, per il quale una cosa è conoscibile «quando si sa indicare tutte le note che prese insieme sono sufficienti per conoscerla e per distinguerla da tutte le altre» (§ 71, p. 63).

4 Cf. al riguardo MITTMANN, 1993, p. 16. 
cui note non ammettono nessun'altra definizione, ma devono essere determinate esaustivamente mediante un principio determinato attraverso se stesso» (RGS 4, p. 57; trad. it. REINHOLD, 2002, p. 117) - cioè il principio di coscienza - , in secondo luogo perché le definizioni che la Filosofia elementare utilizza, contengono concetti che sono adeguati all'oggetto non per mezzo dell'intuizione, come in Kant, ma per mezzo della coscienza. Il concetto della rappresentazione può in tal senso essere definito in maniera completa, in quanto esso «non contiene neppure una nota che sia capace o che abbia bisogno di una spiegazione» (RGS 4, p. 60; trad. it.: REINHOLD, 2002, p. 121). Il tema kantiano della sintesi - che nella conoscenza matematica si realizza mediante intuizioni che mostrano l'adeguatezza della definizione concettuale nell'oggetto e che nella conoscenza filosofica è invece esposto all'insicurezza che il concetto venga davvero rappresentato «in modo dettagliato» (Critica della ragion pura, B 756; trad. it.: KANT, 1986, p. 560) - viene in Reinhold affiancato dalla tesi che la certezza apodittica delle definizioni - prima tra tutte quella del concetto della rappresentazione - possa essere raggiunta anche in filosofia nella misura in cui la corrispondenza tra essa e il suo oggetto è assicurata dal principio della coscienza (Satz des Bewußtseins). In definitiva, poiché il principio della coscienza esprime immediatamente il fatto «autoevidente» della coscienza (Tatsache des Bewußtseins) (RGS 4, p. 62; trad. it.: REINHOLD, 2002, p. 123), la definizione del concetto della rappresentazione, che è parte integrante di quel principio e che costituisce la spiegazione fondamentale (Grunderklärung) dell'intera Filosofia elementare, potrà essere adeguata e completa in quanto si limita a contenere solo le note determinate in maniera originaria dal fatto autoevidente della coscienza. La questione della sintesi kantiana viene qui nominalmente assicurata dal principio di coscienza che sintetizza le parti che lo compongono, di fatto viene tuttavia depotenziata a favore dell'individuazione di concetti che siano semplici in quanto contenenti note ultime e assolutamente determinate. Reinhold può così chiarire che le note del concetto di rappresentazione sono in primo luogo chiare, «dal momento che sono determinate immediatamente attraverso fatti evidenti», che sono in secondo luogo sufficienti «perché tramite esse la rappresentazione può essere distinta da tutto ciò che non è 
rappresentazione», che la definizione della rappresentazione presenta in terzo luogo - proprio come vuole Kant - il concetto entro i suoi limiti, in quanto «in essa non sono contenute note della rappresentazione in numero maggiore di quelle che appartengono al concetto dettagliato della rappresentazione», che tale definizione è in quarto ed ultimo luogo originaria dal momento che non abbisogna di nessun'altra dimostrazione o spiegazione, essendo nient'altro che la scomposizione del principio di coscienza che espone direttamente il fatto autoevidente della coscienza (RGS 4, p. 61; trad. it.: REINHOLD, 2002, p. 121).

\section{Tesi 3: "Fondamento» è in Reinhold la ratio dell'esistere e dell'esser posto di qualcosa. Ciò può darsi, in primo luogo, come fondamento formale mediante il quale una certa sintesi può realizzarsi.}

Reinhold è d'accordo con la definizione del fondamento che Kant dà nella già menzionata lettera del 12 maggio 1789: «(qvo posito determinate ponitur aliud)»(RKA 2, p. 97). Nel primo volume dei Beiträge zur Berichtigung bisheriger Mißverständnisse der Philosophen (1790) egli definisce non a caso il fondamento come ciò «attraverso cui viene posto qualcos'altro in maniera determinata» (REINHOLD, 2003, p. 79). Il modo dell'essere determinato deve per lui essere individuabile con precisione nella misura in cui la ratio che sta alla base di tale determinazione deve riguardare l'ambito del conoscibile. Un fondamento può pertanto essere ciò «a partire dal quale si può comprendere quello che è conoscibile di una cosa» (REINHOLD, 2003, p. 29; trad. it.: REINHOLD, 2002, p. 18). Si tratta dunque di un fondamento che è ratio dell'essere determinato fenomenico.

Una tale definizione del fondamento ha ora due significati differenti nella Filosofia elementare: secondo il primo di essi qualcosa è fondamento dal momento che rappresenta la ratio formalis mediante la quale diventa possibile la conoscenza del fenomeno. Il secondo significato postula invece che qualcosa sia fondamento in quanto costituisce la ratio formalis che realizza la medesima sintesi fenomenica. Si potrebbe al riguardo formulare le due differenti concezioni del fondamento sulla 
base dei concetti di «elemento mediatore» e di «principio della sintesi». Per entrambe vale il già richiamato rapporto intrinseco tra il fondamento e le note costitutive. Da sottolineare rimane tuttavia la loro differenza essenziale che caratterizza nonostante l'origine comune due diversi programmi di fondazione. Laddove nel primo caso il fondamento esercita la funzione di momento mediante il quale la sintesi viene fondata in quanto viene resa possibile nella sua determinatezza, nel secondo caso si tratta di una fondazione nel senso dell'esser posto della sintesi medesima. Solo in quest'ultimo caso è lecito parlare di una coincidenza completa del sostantivo «fondamento» con il verbo «fondare», dal momento che solo qui il fondamento non costituisce soltanto ciò su cui si fonda la sintesi, ma piuttosto ciò che sintetizza in maniera fondativa.

Partiamo dal primo significato secondo il quale il fondamento rappresenta la ratio formalis di una conoscenza sintetica. Reinhold definisce fondamento ultimo ovvero principio della filosofia ciò che viene determinato dalla mera facoltà della rappresentazione (REINHOLD, 2003, p. 46). Gli elementi che fondano qualcosa nella misura in cui lo pongono in maniera determinata, vale a dire lo rappresentano in modo assolutamente necessario, sono le forme universali e necessarie tanto della rappresentazione in generale quanto delle rappresentazioni sensibili, dei concetti e delle idee in particolare. Un oggetto è dunque fondato nel suo esserci e nel suo essere determinato, se lo si rappresenta in modo assolutamente necessario ed universale. Ciò richiede tuttavia di ricorrere a certe forme del rappresentare che fondano la determinatezza degli oggetti. Le forme della facoltà rappresentativa costituiscono pertanto le rationes ultime della filosofia, dal momento che sono il presunto elemento ultimo «a partire dal quale qualcosa è comprensibile» (REINHOLD, 2003; trad. it.: REINHOLD, 2002, p. 42). Nel Corso di Logica esse vengono chiamate fondamenti assolutamente ultimi in quanto «sono le leggi originarie e quindi ultime alle quali è legata la nostra facoltà di rappresentare» (RGS 12, p. 32). All'interno del trascendentalismo della Filosofia elementare le forme della rappresentazione vengono considerate soggettive nella misura in cui appartengono esclusivamente al soggetto distinto dall'oggetto e dalla rappresentazione. Il fondamento può dunque essere compreso come un elemento 
soggettivo dal momento che è "proprietà originaria» dell'elemento rappresentante del fatto della coscienza (REINHOLD, 2003, p. 130). Questo non compromette tuttavia l'oggettività assoluta di ogni necessaria ed universale determinazione del mondo, dal momento che tale oggettività è resa possibile proprio da tali forme soggettive; le forme soggettive della rappresentazione avanzano dunque la pretesa di realizzare, mediante il loro carattere a priori, tali determinazioni dell'oggettualità. Ci troviamo qui di fronte alla traduzione, nei termini della Filosofia elementare, dell'idea che muove l'intera deduzione trascendentale delle categorie kantiana.

Con una differenza fondamentale: alla domanda sul perché le forme della rappresentazione possano essere considerate fondamento del sapere filosofico si deve dunque rispondere che esse sono le determinazioni interne dell'oggettualità. «La forma della rappresentazione è necessariamente presente, non appena è presente una rappresentazione; e poiché un oggetto può essere un rappresentato solo per il fatto che gli viene riferita una rappresentazione, così la forma della rappresentazione è una nota assolutamente necessaria di ogni oggetto, nella misura in cui esso è o può essere un rappresentato» (REINHOLD, 2003, p. 53; trad. it.: REINHOLD, 2002, p. 41-42). Poiché dunque la filosofia non si occupa dell'origine delle rappresentazioni ma delle determinazioni essenziali delle loro componenti - non il «Woraus» ma il «Worin» descrive per Reinhold l'autentico atteggiamento filosofico ${ }^{5}-$, la domanda sul fondamento si riduce al ritrovamento delle note di certi concetti mediante i quali possono essere spiegate in modo determinato altre note o dello stesso concetto o di un altro concetto (nel caso in questione: dell'oggettualità). Le forme della rappresentazione sono dunque i fondamenti ultimi, vale a dire le rationes formales del sapere filosofico, dal momento che rappresentano le note che rendono possibile una relazione fondativa in quanto pongono qualcos'altro in maniera determinata. La questione kantiana della possibilità di una sintesi oggettuale mediante la relazione tra categoria e intuizione cede spazio

5 Su questo punto mi permetto di rimandare a FABBIANELLI, 2011, p. 50-54. 
alla domanda sull'analisi concettuale delle forme rappresentative in quanto note necessarie dell'oggetto.

\section{Tesi 4: «Fondamento» è per Reinhold, in secondo luogo, la ratio dell'esistere e dell'esser posto di qualcosa nel senso del principio sintetico che compie la sintesi stessa.}

Nello scritto contro Eberhard Kant afferma che i giudizi sintetici «non sono possibili in altro modo che sotto la condizione di una intuizione sottesa al concetto del loro soggetto, la quale, se sono giudizi d'esperienza, è empirica, mentre se sono giudizi sintetici a priori è una intuizione pura a priori» (AA 8, p. 241, trad. it.: KANT, 1994, p. 125). La sintesi di predicato e soggetto è possibile secondo la Critica della ragione soltanto nella misura in cui si dà una intuizione. «La Critica però indica questo fondamento di possibilità con chiarezza, e cioè che dev'essere la pura intuizione, sottesa al concetto del soggetto, in rapporto alla quale è possibile, anzi: con la quale soltanto è possibile collegare a priori un predicato sintetico con un concetto» (AA 8, p. 242; trad. it.: KANT, 1994, p. 126). Kant distingue chiaramente tra il fondamento della sintesi a priori - l'intuizione - e la facoltà che compie la stessa azione della sintesi. Nella Critica della ragion pura egli chiama la sintesi «risultato (Wirkung) [...] di una funzione dell'anima, cieca e tuttavia indispensabile», costituita dalla facoltà attiva dell'immaginazione (A 78/B 103; trad. it.: KANT, 1986, p. 144).

La distinzione operata da Kant tra il fondamento della sintesi - l'intuizione - e la facoltà che opera la sintesi - vale a dire l'immaginazione - ritorna anche nella Filosofia elementare: entrambi i momenti vengono tuttavia sussunti sotto la categoria del fondamento. Nella sua recensione del Philosophisches Magazin di Eberhard, Reinhold asserisce che «il fondamento ultimo della sintesi è ciò che è dato nell'intuizione» («Allgemeine Literatur-Zeitung», n. 175, 12.6.1789, Col. 589). Nella Teoria dell'intelletto, che egli formula all'interno della sua Teoria della facoltà della rappresentazione, il fondamento viene invece inteso, oltre che come la forma della rappresentazione quale ratio formalis 
dell'attività sintetica - cioè le categorie come forme delle rappresentazioni dell'intelletto - anche come la facoltà dell'attività sintetica cioè l'intelletto che sintetizza in un certo modo, mediante la propria spontaneità, il molteplice della conoscenza.

Abbiamo così introdotto il sopra annunciato secondo significato di fondamento. Il fondamento è qui il principio della sintesi stessa, cioè quell'elemento che non soltanto rende possibile la sintesi - come in Kant l'intuizione -, ma che opera la sintesi medesima. In tale rispetto, Reinhold parla spesso delle facoltà come dei fondamenti delle connessioni sintetiche che stanno alla base della conoscenza. Che questa seconda determinazione del fondamento non coincida con quella precedente, lo dimostra il fatto che le forme della rappresentazione, che costituiscono le differenti forme dell'unità del molteplice e possono pertanto essere considerate fondamenti nel primo senso, vengono esse stesse prodotte dalla spontaneità della facoltà rappresentativa che è invece centrale per il secondo significato del fondamento (REINHOLD, 2003, p. 131). La facoltà rappresentativa costituisce pertanto il fondamento delle forme della rappresentazione dal momento che ne è il principio produttivo. Per la nostra riflessione è tuttavia importante sottolineare che ciò che vale per la prima determinazione del fondamento vale anche in relazione alla seconda determinazione del medesimo: che cioè la facoltà è un fondamento dal momento che grazie alle sue note costitutive è in grado di spiegare, cioè di porre qualcos'altro in maniera determinata.

Questa seconda accezione del fondamento come ratio che realizza la sintesi fondativa si mostra in maniera evidente negli argomenti con i quali Reinhold difende la propria dottrina della libertà contro Kant. Anche in questo caso, come già rispetto all'atto sintetico della conoscenza, ci troviamo di fronte a due programmi fondativi molto diversi. Laddove in Kant il concetto della libertà viene fondato in base al rapporto intrinseco che lo unisce a quello della legge morale - la legge morale è la ratio cognoscendi della libertà e viceversa la libertà è la ratio essendi della legge morale -, in Reinhold la libertà viene spiegata in relazione alla volontà quale facoltà fondamentale della persona che può autodeterminarsi al soddisfacimento o non soddisfacimento della richiesta del 
desiderio ${ }^{6}$. Anche in questo caso si tratta di una determinazione corretta del concetto di volontà, che Kant avrebbe soltanto preparato ma non prodotto. Kant avrebbe infatti descritto «la volontà ora mediante questa, ora mediante quella nota»; essa sarebbe pertanto di volta in volta stata contrassegnata in maniera sufficientemente determinata solo in relazione alla prospettiva di cui si parla. La stessa nota, utilizzata invece in un rispetto differente, confonderebbe la volontà con altre cose (RGS 2/2, p. 186). Soltanto ponendo in rilievo l'autentica nota della volontà diventa possibile dare una corretta definizione della libertà che la contraddistingue. La dottrina della libertà kantiana deve dunque secondo Reinhold essere respinta, dal momento che confonde la determinazione essenziale, vale a dire la nota ultima che contraddistingue la volontà, con altre note. Ciò avviene ad esempio quando la libertà della volontà viene spiegata come l'esser sottoposto della volontà alla ragion pratica o alla legge morale (RGS 2/2, p. 189). La nota ultima della libertà è secondo Reinhold l'arbitrio, «l'autoattività (Selbstthätigkeit) della persona nel volere» (RGS $2 / 2$, p. 192), la quale rappresenta una nota completamente differente rispetto all'autoattività della ragione. Solo la volontà quale arbitrio può quindi essere compresa come la facoltà che ha il fondamento delle azioni in se stessa. Per Reinhold, andare alla ricerca di fondamenti oggettivi che si trovano presuntivamente al di fuori della volontà significa non avere inteso la natura di questa facoltà fondamentale della persona. Il fondamento ultimo dell'azione libera è dunque la libertà stessa, giacché essa rappresenta «l'ultimo fondamento pensabile dell'azione». La libertà è «la causa assoluta, ultima del proprio agire, oltre la quale non si può andare, visto che essa non dipende veramente da nessun'altra» (RGS 2/2, p. 193). Anche in questo caso, come già per il concetto della rappresentazione, è l'autocoscienza a dirci che ci troviamo di fronte al fondamento ultimo: la libertà della volontà è un fatto (Tatsache) di cui il sapere filosofico pone in evidenza le note ultime attraverso un'analisi concettuale.

6 Su questo punto si vedano, tra gli altri, FABBIANELLI, 2000; LAZZARI, 2003; BONDELI, 2012. 


\section{Tesi 5: Fondamento è, in terzo luogo, il fundamentum ovvero la base su cui poggia l'intero edificio del sapere.}

Accanto al fondamento come ratio nel duplice rispetto di elemento mediatore e di principio della sintesi si trova in Reinhold un terzo significato di fondamento: il fundamentum ovvero la base su cui si fonda qualcosa. Sarà proprio questo senso del fondamento a rilevarsi prevalente all'interno dei grandi sistemi della filosofia classica tedesca. La variazione semantica del concetto di fondamento la si comprende tenendo presente che le già menzionate forme della rappresentazione costituiscono i principi ultimi, le rationes ultimae non in generale, ma soltanto rispetto agli oggetti. Considerate in sé, esse non sono infatti né autoesplicative né autofondative, devono piuttosto essere dedotte dalla coscienza (REINHOLD, 2003, p. 59). Il fondamento (Grund) nel senso del fondamento (Fundament) del sapere filosofico risiede pertanto nel fatto della coscienza: «La coscienza è l'autentico fondamento (Grund) ultimo, il fondamento (Fundament) al quale viene riportata la teoria della facoltà della rappresentazione» (REINHOLD, 2003, p. 194).

Che il fatto della coscienza costituisca la vera e propria base della Filosofia elementare lo si riconosce dal suo essere non soltanto universalmente valido (allgemeingültig), ma anche compreso come universalmente valido (allgemeingeltend). Si tenga presente questa importante distinzione: «Il principio universalmente compreso come valido si distingue in filosofia da quello universalmente valido per il fatto che non solo viene trovato, come questo, vero da ciascuno che lo comprende, ma per il fatto che viene anche effettivamente compreso da ogni mente sana e filosofante» (RGS 1, p. 70; trad. it.: REINHOLD, 2006, p. 65)7. Si noti anche che qui si tratta di un principio (Prinzip) che deve essere espresso filosoficamente attraverso una proposizione fondamentale (Grundsatz) che sia assolutamente prima; il suo carattere principiale non coincide dunque necessariamente con il suo carattere di proposizionalità. Ciò significa che il principio (Prinzip) assoluto della filosofia è una proposizione fondamentale (Grundsatz) che è assolutamente evidente nella

7 Cf. al riguardo BONDELI, 1993, p. 102-107. 
misura in cui esprime il fondamento (Fundament) del sapere in generale costituito dalla coscienza. L'evidenza assolutamente ultima appartiene perciò primariamente non ad una proposizione, ma al fatto che le sta a fondamento. Se i concetti del «principio di coscienza» (Satz des Bewußtseins) - rappresentazione, soggetto e oggetto - non fossero determinati mediante il fatto della coscienza, non potrebbero avanzare la pretesa di essere assolutamente validi ed assolutamente compresi come validi. E la stessa cosa varrebbe anche per il principio di coscienza che si costituisce mediante essi.

La giustificazione filosofica della coscienza come fondamento (Fundament) è la medesima di quella apportata da Reinhold per le forme della rappresentazione ovvero per le facoltà quali rationes della filosofia: essere composta di parti le cui note non possono essere fatte derivare da altro in quanto sono originarie. Le determinazioni della rappresentazione, del soggetto e dell'oggetto - quali momenti che costituiscono la coscienza - dipendono infatti dal loro scaturire immediatamente («quillen») dalla coscienza medesima (REINHOLD, 2003, p. 113). Questa loro originarietà assoluta garantisce che siano autoevidenti. Se le note delle componenti della coscienza non fossero le ultime, conterrebbero altre note eruibili solo grazie ad una scomposizione delle prime. Ne segue, come già si osservava in precedenza, che mentre il sapere matematico è evidente per il fatto che costruisce i propri oggetti mediante l'intuizione, il sapere filosofico raggiunge l'evidenza solo quando è in grado di rintracciare le note «che non sono ulteriormente scomponibili, che proprio per questo sono chiare di per sé (einleuchten) e devono essere rappresentate da ogni pensatore allo stesso modo, essere comprese da tutti come valide» (REINHOLD, 2003, p. 91-92). Proprio questo è il caso delle note delle parti che compongono la coscienza: sono note evidenti e comprese universalmente come valide in quanto sono completamente (durchgängig) determinate. Si badi bene che la loro determinazione completa rappresenta una qualità prefilosofica che spetta loro prima di ogni riflessione scientifica. Come tali, devono essere considerate quali note che non sono il risultato di un'astrazione 
(Abstraktion) ma piuttosto di una riflessione (Reflexion $)^{8}$ sulla coscienza medesima. Esse «non presuppongono pertanto nessun ragionamento (Raisonnement) e precedono ogni filosofia» (REINHOLD, 2003, p. 113). Se così non fosse, non si potrebbe affatto distinguere, come invece fa Reinhold, tra due forme di evidenza: da un lato, l'evidenza scientifica, dall'altro lato l'evidenza che le sta a fondamento e che si trova «al di fuori dell'intero ambito della scienza». A questa duplice forma di evidenza corrispondono due fonti differenti: il principio di coscienza, quale fondamento (Grund) «dell'intera evidenza scientifica», e il fatto della coscienza, quale fondamento (Grund nel senso di Fundament) di tutta l'evidenza in generale (REINHOLD, 2003, p. 196). Reinhold può dunque affermare che le note delle parti che compongono la coscienza possono essere pensate come completamente determinate e pertanto come evidenti, prima che la speculazione scientifica le consideri come i momenti comuni contrassegnabili mediante il concetto della specie (REINHOLD, 2003, p. 114). Quando si legge nel primo volume dei Beiträge che un fondamento (Grund) universalmente compreso come valido "può passare nell'ambito della filosofia solo nella misura in cui diventa una proposizione fondamentale (Grundsatz)» (REINHOLD, 2003, p. 98), ciò non significa che i fondamenti universalmente compresi come validi siano rinvenibili unicamente all'interno del pensiero filosofico. Vuol dire al contrario che la trasformazione della validità prescientifica di un fondamento nella sua validità scientifica esige che il fondamento si trasformi in una proposizione fondamentale. Solo in quanto una tale trasformazione avviene effettivamente - e ciò si verifica nella scienza -, solo in tanto è giustificabile distinguere tra fondamenti differenti ponendoli in una classificazione per la quale si danno fondamenti assoluti e relativi, fondamenti primi e derivati.

Quanto appena detto possiamo riassumerlo nella seguente maniera: il fondamento (Grund come Fundament) del sapere in generale, e pertanto anche del sapere filosofico in particolare, è la coscienza, dal momento che essa si compone di parti assolutamente semplici, non ulteriormente analizzabili e proprio perciò evidenti. Una tale qualità del

8 Cf. al riguardo BREAZEALE, 2005. 
fondamento spetta però alla coscienza in sé, vale a dire alla coscienza considerata prima di ogni speculazione.

\section{Tesi 6: La dottrina del fondamento reinholdiana spiega in che senso la Filosofia elementare possa avanzare la pretesa di andare oltre la Critica della ragione kantiana; come tale, essa rappresenta un punto di vista privilegiato per comprendere la genesi della filosofia classica tedesca.}

A partire dal 1790 Reinhold non perde occasione per mettere in risalto la distanza che a suo avviso separa la Filosofia elementare dalla Critica della ragione kantiana. Egli ritiene che in relazione al concetto del fondamento nel senso di Fundament la filosofia kantiana si appoggi all'esperienza, che la Filosofia elementare faccia invece riferimento alla coscienza. Per Reinhold, Kant pone la questione della possibilità dei giudizi sintetici a priori, dal momento che intende mostrare che l'esperienza consiste nella connessione necessaria delle percezioni. Kant prende pertanto le mosse da una precisa concezione del concetto di esperienza che nella Critica della ragion pura viene definito nei termini della «rappresentazione del nesso necessario degli oggetti delle rappresentazioni sensibili» e che costituisce la vera e propria base dell'intero edificio del sapere kantiano (REINHOLD, 2003, p. 198).

Reinhold è dell'avviso che l'esperienza non possa rappresentare il fondamento del sapere filosofico, in quanto le note che secondo Kant dovrebbero contrassegnarla - la necessità e l'universalità - le spettano soltanto in un senso derivato. Egli interpreta dunque il dissidio tra Kant e Eberhard, al quale egli stesso partecipa, come la prova indiretta del fatto che il concetto dell'esperienza operante nella Critica della ragione non è stato sufficientemente determinato e che pertanto non viene ancora compreso da tutti come valido. Kant sviluppa certamente le note essenziali del concetto di esperienza, ad esempio nella deduzione delle categorie, lo fa tuttavia partendo dal presupposto che il concetto dell'esperienza debba essere ammesso «come certo senza alcuna prova» (REINHOLD, 2003, p. 226). 
L'obiezione di Reinhold è al contempo chiara ed incisiva: l'esperienza della Critica della ragione speculativa non può essere considerata come l'autentico fondamento (Grund come Fundament) della filosofia in quanto conduce ad una fondazione circolare. Le note che la contrassegnano - la necessità e l'universalità - vengono infatti determinate e pertanto giustificate proprio a partire dalla premessa che possa essere riconosciuta una validità assoluta al concetto dell'esperienza che le esprime. Sulla base dell'esperienza esse non possono tuttavia essere giustificate come comprese da tutti come valide: non dal punto di vista fattuale, dal momento che non sono ancora state in grado di risolvere tutti i fraintendimenti verificatisi nella discussioni filosofiche; non dal punto di vista teorico, giacché esse possono essere giustificate e spiegate dall'altra fonte di legittimazione costituita dalla coscienza. Reinhold può dunque affermare che la Teoria della facoltà della rappresentazione non prende le mosse dal concetto del giudizio sintetico, «di cui non ha bisogno fino a quando il medesimo non sia stato completamente sviluppato da premesse da lui del tutto indipendenti» (REINHOLD, 2003, p. 203). Reinhold è sì d'accordo con Kant che il sapere filosofico sia sintetico a priori, egli ritiene tuttavia che la domanda kantiana sui giudizi sintetici a priori non debba essere la domanda prioritaria all'interno della Filosofia elementare, dal momento che può essere considerata come «una conseguenza (Folge)» ovvero «come una spiegazione (Erläuterung)» della vera domanda filosofica: non si chiede come siano possibili i giudizi sintetici a priori, ma invece come sia possibile la coscienza (REINHOLD, 2003, p. 194). Anche la coscienza consiste nella duplice sintesi a priori che si specifica nei due momenti costitutivi della distinzione della rappresentazione dal soggetto e dall'oggetto ovvero della sua relazione ad essi. Una tale duplice sintesi sarebbe tuttavia superiore a quella kantiana dal momento che concerne fatti autoevidenti. E questi fatti sono autoevidenti in quanto sono contrassegnati da note assolutamente ultime che l'analisi concettuale della filosofia deve portare ad evidenza scientifica.

È in questo contesto critico che la nota della necessità (ma lo stesso discorso può essere fatto valere in relazione alla universalità) viene da Reinhold abbassata a determinazione derivata e, come tale, non 
autosufficiente, nel senso che può essere fatta derivare dalla priorità (apriorità) delle forme della rappresentazione. «Si mostra infatti che la coscienza in generale sarebbe impossibile se la prodotta unità del molteplice dato, nella quale consiste la forma della rappresentazione in generale, non fosse determinata nella mera facoltà della rappresentazione» (REINHOLD, 2003, p. 194). Solo la coscienza può dunque costituire il vero fondamento della filosofia, dal momento che solo essa rappresenta un fatto assolutamente evidente. E il fatto della coscienza è assolutamente evidente tanto in relazione al suo contenuto, visto che i concetti che lo compongono non si lasciano scomporre ulteriormente e sono come tali completamente determinati, quanto rispetto alla forma, poiché le proposizioni che la esprimono sono tra loro connesse in maniera rigorosamente sistematica (RGS 4, p. 65).

La Filosofia elementare di Reinhold rappresenta in modo evidente un programma filosofico alternativo a quello della Critica della ragione kantiana. Essa ne è consapevole e lo rivendica in maniera esplicita. I passi nei quali una tale distanza si mostra chiaramente sono noti: nella Fundamentschrift si legge ad esempio che Kant mirava a spiegare come gli oggetti potessero diventare conoscibili sulla base dell'intuizione (RGS 4, p. 59), che dunque il suo problema era esclusivamente «la possibilità della scienza degli oggetti in senso proprio» (RGS 4, p. 43, trad. it.: REINHOLD, 2002, p. 101), vale a dire la possibilità della metafisica dei fenomeni. Proprio perciò - questa la conclusione di Reinhold la Critica della ragione kantiana può soltanto essere considerata come una propedeutica (Propädeutik) dell'autentica Filosofia senza appellativi, cioè della Filosofia elementare, che invece avanza la pretesa di essere scienza del rappresentare in generale, dal quale dipende tanto il conoscere quanto l'appetire (RGS 4, p. 47).

Ritengo che la trasformazione del concetto di fondamento che avviene nel passaggio dal progetto kantiano della Critica della ragione al progetto reinholdiano della Filosofia senza appellativi possa essere considerata come un punto di osservazione privilegiato dal quale indagare l'origine e lo sviluppo della filosofia classica tedesca. A conclusione delle mie riflessioni, e soltanto per introdurre brevemente un tema che dovrebbe essere spiegato in maniera molto più ampia, vorrei fare 
alcune brevi annotazioni sul nuovo statuto del concetto del fondamento all'interno della Dottrina della scienza di Fichte che, come è noto, si riallaccia esplicitamente alla Filosofia elementare di Reinhold.

Il triplice carattere del concetto del fondamento reinholdiano in primo luogo come la ratio della sintesi, in secondo luogo come lo stesso principio sintetizzante, infine come la base (Fundament) di tutte le azioni sintetiche - rappresenta il punto da cui muove Fichte nella propria concezione del fondamento. Il passaggio al sistema della Dottrina della scienza si realizza - potremmo anche dire - proprio attraverso la riduzione dei tre significati individuabili in Reinhold ad un'unica determinazione che li collega in una maniera peculiare. Il fondamento in senso fichtiano diventa in altre parole ciò in cui la ratio nella duplice accezione reinholdiana e il fundamentum della Filosofia elementare si uniscono. Rilevante è che ciò vada di pari passo con la trasformazione della natura dell'attività che viene attribuita al fondamento, non più soltanto meramente sintetica ma tetica.

Il Fondamento dell'intera dottrina della scienza spiega al riguardo che l'Io assoluto rappresenta il fondamento (Grund) ultimo della filosofia nella misura in cui la sua attività coincide con il suo essere. "L'Io è e pone il suo essere in forza del suo puro e semplice essere. - Esso è nel contempo l'agente e il prodotto dell'azione, ciò che è attivo e ciò che è prodotto dall'attività; azione e atto sono un'unica e medesima cosa» (GA I 2, p. 259; trad. it.: FICHTE, 2017, p. 149). L'Io è pertanto autofondamento (Selbstgrund), cioè causa sui, dal momento che è la ratio del suo proprio agire. Questo agire ha ora il proprio fondamento in sé e non al di fuori di sé, in quanto il suo principio (Prinzip) è proprio quell'essere dell'Io che agisce. Nella misura in cui è autofondativo l'Io rappresenta però anche l'assoluto fondamento (Fundament) del sapere. Il fondamento (Grund) assume allo stesso tempo tanto la funzione di ratio quanto quella di fundamentum. Alla contestuale eliminazione della differenza ancora presente in Reinhold tra i due momenti - le rationes sono costituite nella Filosofia elementare o dalle forme della rappresentazione o dalle facoltà, mentre invece il fundamentum è rappresentato dalla coscienza - corrisponde nella Dottrina della scienza la sostituzione dell'attività sintetica con l'attività tetica. Poiché non si 
pone originariamente né come uguale (sintesi) né come contrapposto (antitesi) a qualcosa ma pone soltanto se stesso (tesi), il fondamento deve assumere le funzioni tanto della ratio dell'agire quanto del fundamentum su cui tale agire riposa.

È importante osservare che Fichte non confuta semplicemente la concezione del fondamento reinholdiana, ma la inserisce nel proprio nuovo schema speculativo. Lo si può vedere facendo riferimento a quattro punti. 1) Il primo principio della Dottrina della scienza può essere considerato come il principio assolutamente incondizionato in quanto esprime quell'atto (Tathandlung) che rappresenta il fondamento (Grundlage) del sapere umano. Un tale rapporto riprende e modifica al contempo la relazione tra il principio del sapere filosofico e il fatto della coscienza di cui parla la Filosofia elementare. 2) Fichte è d'accordo con Reinhold che l'esposizione filosofica dell'atto originario quale fondamento (Grund) assoluto sia corretta nella misura in cui vi si pensa ciò che in esso è effettivamente contenuto e non vi si pensa «quanto non si deve pensare» (GA I 2, p. 255; trad. it.: FICHTE, 2017, p. 139). 3) A tale scopo è necessaria un'astrazione filosofica che svolge il ruolo che nella Filosofia elementare aveva la riflessione sulla coscienza. 4) Anche Fichte, come già Reinhold, pensa tuttavia che si possa compiere una determinazione corretta del fondamento assoluto solo in quanto si è in grado di trovare la nota essenziale che lo caratterizza. L'Io può dunque essere considerato quale fondamento (Grund) ultimo della filosofia in quanto si conosce il suo «carattere» (Charakter) (GA I 2, p. 258). E il suo carattere consiste nell'essere una attività che si pone in maniera incondizionata e che si fonda su se stessa.

\section{Bibliografia}

BONDELI, M. Das Anfangsproblem bei Karl Leonhard Reinhold: Eine systematische und entwicklungsgeschichtliche Untersuchung zur Philosophie Reinholds in der Zeit von 1789 bis 1803. Frankfurt a. M: Klostermann, 1993. 
BONDELI, M. Zu Reinholds Auffassung von Willensfreiheit in den Briefen II. In: V. Stolz et al. (Hg.): Wille, Willkür, Freiheit. Reinholds Freiheitskonzeption im Kontext der Philosophie des 18. Jahrhunderts. Berlin: De Gruyter, 2012. p. 125-152.

BREAZEALE, D. Reflection, intellectual intuition, and the fact of consciousness: remarks on the method of Reinhold's Elementarphilosophie (1789-1791). Archivio di filosofia, p. 35-58, 2005.

FABBIANELLI, F. Die Theorie der Willensfreiheit in den „Briefen über die Kantische Philosophie" (1790-92) von Karl Leonhard Reinhold. Philosophisches Jahrbuch, p. 428-443, 2000.

FABBIANELLI, F. Coscienza e libertà: Un saggio su Reinhold. Pisa: Edizioni della Normale, 2011.

FICHTE, J. G. Fichte-Gesamtausgabe der Bayerischen Akademie der Wissenschaften. Herausgegeben von R. Lauth et al. Stuttgart-Bad Cannstatt: FrommannHolzboog, 1962 et seq. (con indicazione del volume). (= GA).

FICHTE, J. G. Fondamento dell'intera dottrina della scienza. A cura di G. Boffi. Milano: Bompiani, 2017.

KANT, I. Critica della ragion pura. A cura di P. Chiodi. Torino: Utet, 1986.

KANT, I. Contro Eberhard: La polemica sulla Critica della ragion pura. A cura di C. La Rocca. Pisa: Giardini Editori e Stampatori, 1994.

KANT, I. Kant's gesammelte Schriften. Herausgegeben. von der Königlich Preußischen Akademie der Wissenschaften. Berlin: De Gruyter, 1900 et seq. (con indicazione del volume). (= AA).

LAZZARI, A. K. L. Reinholds Behandlung der Willens- und Freiheitsthematik zwischen 1789 und 1792. In: BONDELI, M.; SCHRADER, W. H. (Hg.). Die Philosophie Karl Leonhard Reinholds. Amsterdam: Rodopi, 2003. p. 191-215.

MITTMANN, J.-P. Das Prinzip der Selbstgewißheit: Fichte und die Entwicklung der nachkantischen Grundsatzphilosophie. Bodenheim: Athenäum Hain Hanstein, 1993. 
REIMARUS, H. S. Die Vernunftlehre, als eine Anweisung zum richtigen Gebrauche der Vernunft in dem Erkenntniß der Wahrheit, aus zwoen ganz natürlichen Regeln der Einstimmung und des Widerspruchs hergeleitet. Hamburg: Bohn, 1766.

REINHOLD, K. L. Concetto e fondamento della filosofia. A cura di F. Fabbianelli. Roma: Edizioni di Storia e Letteratura, 2002.

REINHOLD, K. L. Beiträge zur Berichtigung bisheriger Mißverständnisse der Philosophen. Erster Band, das Fundament der Elementarphilosophie betreffend. Mit einer Einleitung und Anmerkungen herausgegeben von F. Fabbianelli. Hamburg: Meiner, 2003.

REINHOLD, K. L. Saggio di una nuova teoria della facoltà umana della rappresentazione. A cura di Faustino Fabbianelli. Firenze: Le Lettere, 2006.

REINHOLD, K. L. Gesammelte Schriften. Kommentierte Ausgabe. Herausgegeben von M. Bondeli. Basel: Schwabe, 2007 et seq. (con indicazione del volume). (= RGS).

REINHOLD, K. L. Korrespondenzausgabe der Österreichischen Akademie der Wissenschaften.BegründetvonR.Lauth,K.HillerundW.H.Schrader.Herausgegeben von F. Fabbianelli, K. Hiller und I. Radrizzani. Stuttgart-Bad Cannstatt: FrommannHolzboog, 1983 et seq. (con indicazione del volume). (= RKA).

TEICHNER, W. Rekonstruktion oder Reproduktion des Grundes: Die Begründung der Philosophie als Wissenschaft durch Kant und Reinhold. Bonn: Bouvier, 1976.

Ricevuto: $23 / 08 / 2018$

Received: 08/23/2018]

Recebido: 23/08/2018

Approvato: 20/09/2018

Approved: 09/20/2018 Aprovado: 20/09/2018 